\title{
JASP (Software)
}

JASP, Jeffrey's Amazing Statistics Program (official webpage: https://jasp-stats.org/), version 0.12 .2 , is an open-source, free statistical software package available on Windows, MacOS, and Linux platforms. It was developed by a group of quantitative methodologists who are interested in improving statistical testing and analysis methods used in the fields of psychological sciences. It implements various analysis methods, particularly those in Bayesian analysis and data science. The implemented methods include $t$-tests, ANOVA, regression analysis, factor analysis, machine learning, meta-analysis, network analysis, and SEM. JASP also includes a data editor for visual inspection and pre-processing. It supports data importing and exporting from/to various data sources. The statistical analysis program R constitutes the basis of JASP, but for users who are not familiar with programming, JASP is featured with a graphical user interface (GUI) so that the users can select analysis modules and modify options conveniently. To support users in academia, especially those in psychology, JASP provides functionalities for visualizing analysis results in tables and figures according to the American Psychological Association (APA) convention and presents options for modification once they are created.

\section{Implemented Functionalities}

\section{Basic Statistical Methods}

Although JASP implements various analysis methods, it has been specialized in Bayesian methods. However, since its first version, JASP has provided options to perform both frequentist and Bayesian statistical analysis including $t$-tests, ANOVA (including ANCOVA, MANOVA, and repeated-measures ANOVA), and regression analysis (including linear and logistic regression). JASP reports both the posterior distribution of the parameter of interest (with a Bayesian credible interval) and Bayes factor when Bayesian statistical testing is performed. 
Analysis results are presented in tables or figures in the APA convention following options that can be modified. JASP can also generate additional plots for Bayesian analysis, such as a prior and posterior distribution plot and a Bayes factor robustness check report plot (see Figure 1). As all functionalities are implemented with a GUI, users can perform their intended analysis simply by clicking and using drag-and-drop functions without coding a script, as they can with SPSS or other GUI-aided software packages.

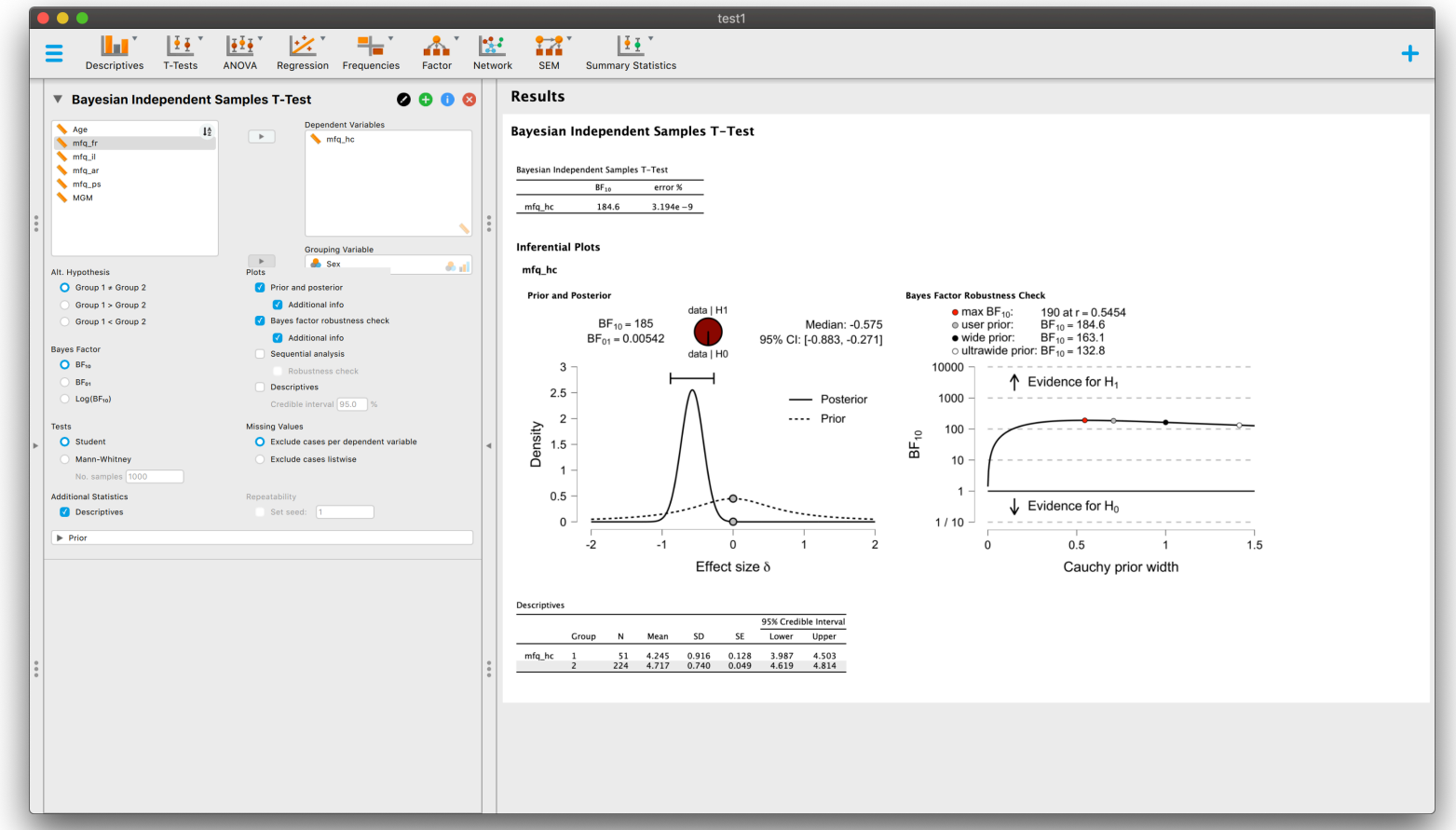

Figure 1. Bayesian analysis results reported by JASP including a prior and posterior distribution plot and a Bayes factor robustness check report plot.

Source: author created this screenshot of JASP for this entry.

\section{Advanced Statistical and Data Scientific Methods}

JASP (version 0.12.2) also supports advanced statistical methods for structural equation modeling (SEM), psychometrics, and statistical testing with frequency data. SEM can be performed with a user generated lavaan syntax. Mediation analysis can also be conducted while 
predictors, mediators, outcome variables, and confounders can be specified with a GUI. Users can perform functionalities in psychometrics such as a reliability check, principal component analysis, and exploratory and confirmatory factor analysis. Diverse frequency testing methods, such as binomial and multinomial regression, log-linear regression, and contingency table analysis, are also supported for both frequentist and Bayesian analysis.

In addition to statistical testing methods that have been frequently used in academia, JASP has functionalities that implement methods in data science (see Figure 2). Network analysis is one of such methods that are featured in JASP. The network analysis implemented in JASP enables users to examine relations between discrete entities in a dataset based on the network theory. It generates diverse forms of reports including visualized network, centrality, clustering plots, and centrality, clustering, and weights matrix tables. Machine learning functionalities have also been implemented in JASP, including regression, classification, and clustering analysis. These methods are implemented with various algorithms, such as boosting, K-nearest neighbors, random forest, and regularization (with both L1 and L2 regularization for Lasso, Ridge, and Elastic-net regression), etc. The machine learning process can be performed with a crossvalidation by default. 


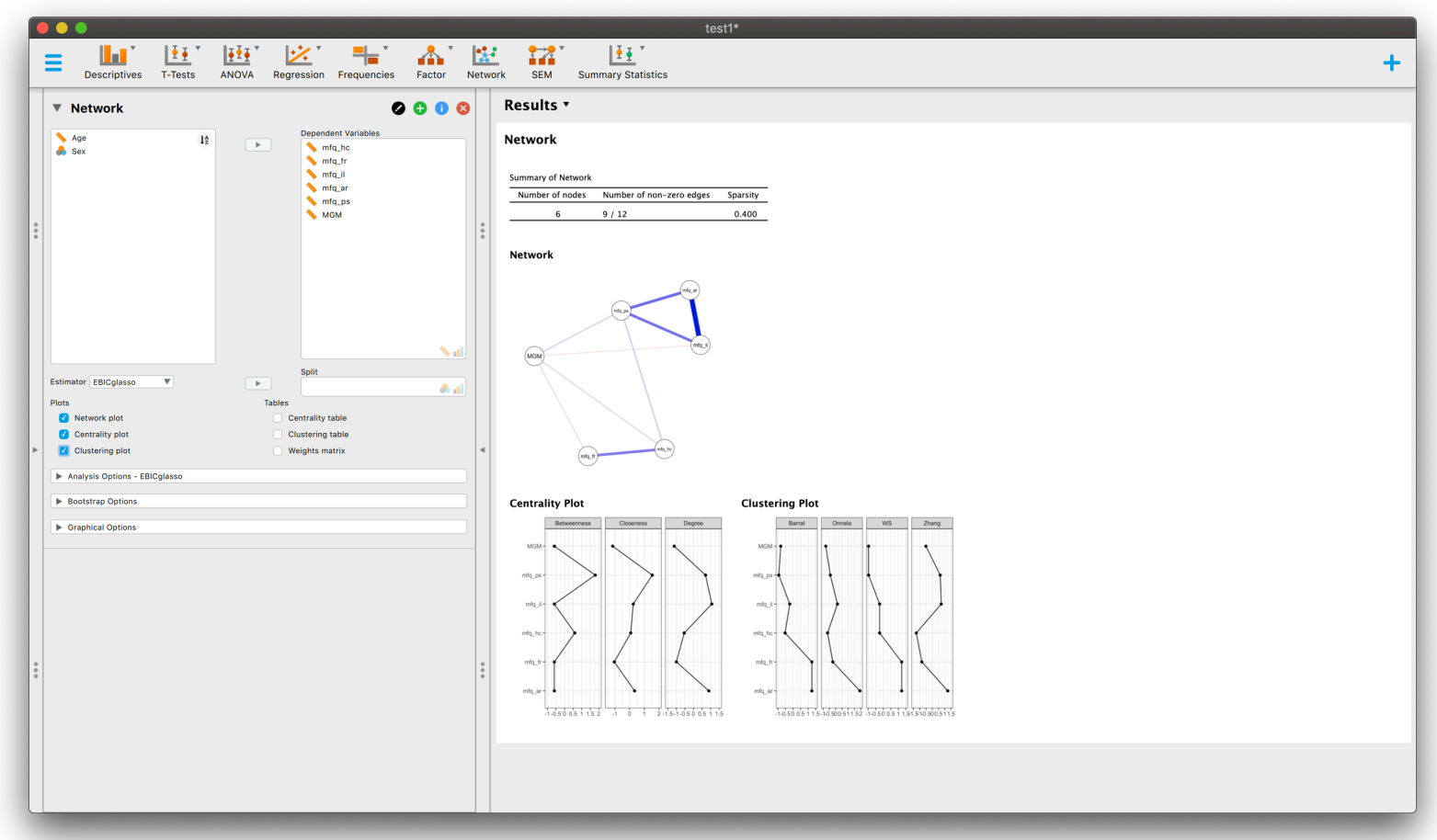

Figure 2. Network analysis implemented in JASP.

Source: author created this screenshot of JASP for this entry.

\section{Data Editing, Importing, and Exporting}

To assist data preprocessing, JASP is featured with a simple data editor that allows users to visually inspect and preprocess their data (see Figure 3). JASP primarily supports a widely used file format, csv. Users can also import data directly from Open Science Framework (OSF) projects or JASP Data Library that contains datasets for tutorials. Once a dataset is imported, it is presented in JASP's data editor for further preprocessing. The viewer, which is similar to a spreadsheet editor, enables users to edit values (through a system default spreadsheet editing program) and filter the imported dataset according to user-defined conditions. Unlike the simple filtering function implemented in ordinary spreadsheet editors, JASP allows users to customize the filtering condition with diverse arithmetic, logical, and statistical functions. Analysis results can be exported in html or directly to OSF projects. JASP can also generate LaTeX codes to 
export created tables. Created tables or figures can also be directly copied to a clipboard. In addition to these options to export analysis results, a dataset being analyzed can be exported in csv or to OSF projects.

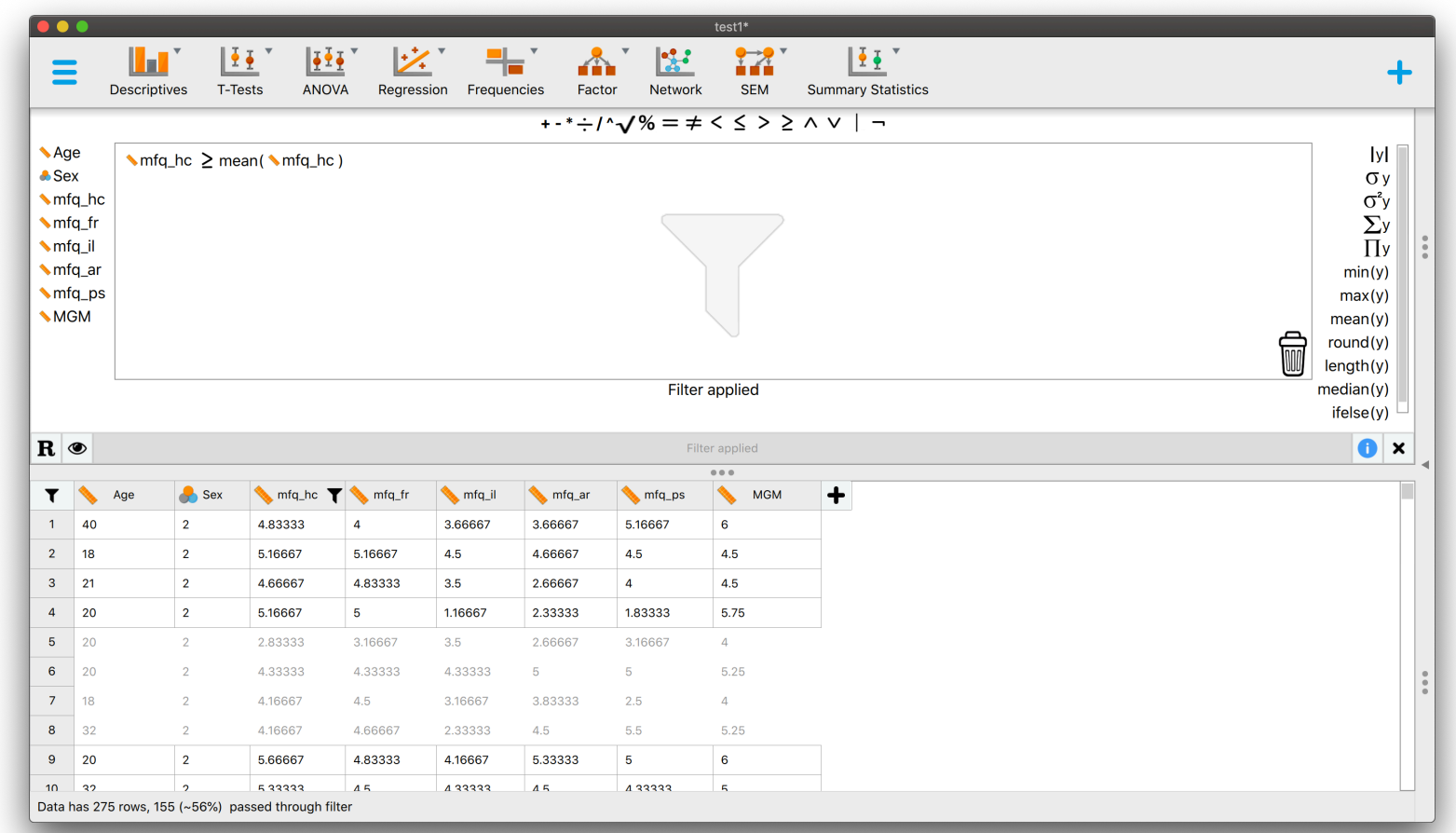

Figure 3. JASP data editor. A statistical filter is applied.

Source: author created this screenshot of JASP for this entry.

\section{Notable Features}

The most notable feature of JASP is that it is available for free for users while implementing diverse functionalities including methods in Bayesian statistics and data science with a GUI. The following is a brief list of analysis methods implemented in JASP: descriptive statistics presentation, frequentist and Bayesian $t$-tests, ANOVA, regression analysis, factor analysis, machine learning, meta-analysis, network analysis, and SEM. Another benefit of using JASP for researchers in academia is that it supports visualizing analysis results in the APA convention. For users who are interested in open science, JASP provides additional functionalities, such as 
importing from, exporting to, and synchronizing with an OSF project space, that allow them to conveniently synchronize the current JASP project with the OSF.

\section{Hyemin Han and Kelsie J. Dawson}

See also Bayesian Data Analysis; R; SPSS

\section{Further readings}

Han, H., Park, J., \& Thoma, S. J. (2018). Why do we need to employ Bayesian statistics and how can we employ it in studies of moral education?: With practical guidelines to use JASP for educators and researchers. Journal of Moral Education, 47(4), 519-537. doi: $10.1080 / 17588928.2019 .1570103$

Love, J., Selker, R., Marsman, M., Jamil, T., Dropmann, D., Verhagen, J., ... \& Matzke, D. (2019). JASP: graphical statistical software for common statistical designs. Journal of Statistical Software, 88(2). doi: 10.18637/jss.v088.i02

Wagenmakers, E. J., Love, J., Marsman, M., Jamil, T., Ly, A., Verhagen, J., ... \& Meerhoff, F. (2018). Bayesian inference for psychology. Part II: Example applications with JASP. Psychonomic Bulletin \& Review, 25(1), 58-76. doi: 10.3758/s13423-017-1323-7 